\title{
Beta-Blocker Therapy Early After Myocardial Infarction: A Comparison Between Medication at Hospital Discharge and Subsequent Pharmacy-Dispensed Medication
}

\author{
Susanne Bendesgaard Pedersen ${ }^{1} \cdot$ Jens Cosedis Nielsen ${ }^{1} \cdot$ Hans Erik Bøtker $^{1} \cdot$ \\ Jeffrey J. Goldberger ${ }^{2}$
}

Published online: 16 June 2016

(c) The Author(s) 2016. This article is published with open access at Springerlink.com

\begin{abstract}
Background Beta-blocker (BB) therapy after myocardial infarction (MI) reduces all-cause mortality.

Objective The aim of this study was to investigate BB dosing patterns and compliance following MI.

Methods Using medical patient files and nationwide databases, we identified 100 patients who were discharged following MI in 2012 from Aarhus University Hospital, Denmark, and subsequently redeemed one or more BB prescriptions within 6 months. We obtained information about all BB medication prescribed at discharge and all BB prescriptions redeemed until 31 December 2013. Daily BB doses were computed as percentages of the target doses used in clinical trials documenting the efficacy of BBs after MI. Four dose groups were defined: $\leq 12.5,>12.5-25,>25-50$, and $>50 \%$ of target dose. The proportion of patients in each dose group was ascertained at and following discharge, as was the proportion that changed dose group following discharge.

Results The median study period was 400 days (interquartile range [IQR] 318-486 days). At discharge, $8 \%$ of daily doses were $>50 \%$ of target dose while $80 \%$ were $\leq 25 \%$ of target dose. At first prescription redemption, $71.7 \%$ of patients moved to a higher dose group (median dose change $=33.4 \%$ [IQR 2.0-115.1]). Still,
\end{abstract}

Electronic supplementary material The online version of this article (doi:10.1007/s40801-016-0079-0) contains supplementary material, which is available to authorized users.

Susanne Bendesgaard Pedersen

susapese@rm.dk

1 Department of Cardiology, Aarhus University Hospital, Palle Juul-Jensens Boulevard 99, 8200 Aarhus N, Denmark

2 Division of Cardiology, Department of Medicine, University of Miami Miller School of Medicine, 1400 N.W. 12th Ave, Miami, FL 33136, USA comparing final daily doses to discharge doses, $40.2 \%$ did not change dose group (median dose change $-5.7 \%$ [IQR -18.0 to 4.2$]$ ). Only $31.5 \%$ reached a final daily dose $>50 \%$ of target dose.

Conclusions Target dose BB treatment was infrequently achieved at discharge following MI. Despite dose up-titration early after discharge, most patients did not receive target dose BB treatment approximately 1 year following MI.

\section{Key Points}

Even though the beneficial effect of beta-blockers was discovered several decades ago, target dose beta-blocker treatment is still infrequently achieved at discharge following myocardial infarction.

Based on redeemed prescriptions rather than selfreported beta-blocker use, this study showed that most patients do not receive target dose beta-blocker treatment approximately 1 year following myocardial infarction.

As beta-blocker dose may affect outcome, our findings emphasize the importance of tracking betablocker use following myocardial infarction to properly assess the impact of treatment and dose on survival.

\section{Introduction}

Beta-blocker (BB) therapy after myocardial infarction (MI) reduces all-cause mortality [1-3] and is recommended by international guidelines in the absence of 
contraindications [4, 5]. Evidence from randomized clinical trials indicate that the target doses of frequently used BBs are metoprolol $200 \mathrm{mg} /$ day [6], carvedilol $50 \mathrm{mg} /$ day [7], bisoprolol $10 \mathrm{mg} /$ day [8], atenolol $100 \mathrm{mg} /$ day [9], and propranolol $180 \mathrm{mg} /$ day [10]. Still, BB doses prescribed for patients after MI are often considerably lower $[11,12]$. Although treatment with doses lower than those used in the randomized clinical trials that established their efficacy might be expected to be associated with poorer outcomes, the PACE-MI (Pacemaker and beta-blocker therapy post-MI) Registry Study-OBTAIN (Outcomes of Beta-blocker Therapy After Myocardial Infarction) recently demonstrated that treatment with target doses was not superior to treatment with $25 \%$ of the target dose [13]. As this is the only available large-scale study to evaluate the effect of BB dose on outcome, further efforts to assess the BB dose-dependency on outcome after MI are required. Large healthcare system databases can be useful to address this question, but are often limited to discharge medication doses. Discharge BB doses may not reflect actual doses taken by patients during follow-up because the prescription was never redeemed or the dose was changed. Non-adherence to prescribed cardiovascular medication is a well known occurrence among post-MI patients, encompassing failure to take the medication as prescribed and failure to take the medication at all $[14,15]$. Thus, some post-MI patients may take BB doses that differ from doses prescribed at discharge. Most previous studies of BB use early after MI relied on prescribed medication or patient recollection of administered medication [11-14], which carries a risk of recall bias. The aim of our study was to compare BB medication prescribed at discharge after MI with subsequent pharmacy-dispensed BB medication, thereby eliminating recall bias. Assuming that pharmacy redemptions are only performed when patients need additional medication, this provides the most accurate assessment of the actual BB consumption for an individual patient.

\section{Methods}

\subsection{Design and Setting}

This observational study was conducted in Denmark where healthcare is tax-funded, guaranteeing all inhabitants access to general practitioners and hospitals [16], and providing partial reimbursement of most prescription medication expenses [17]. Each Danish inhabitant has a unique civil registration number, which is a prerequisite for receiving health care and allows for accurate and unambiguous linkage of national registries [16].

\subsection{The Danish National Patient Registry}

The Danish National Patient Registry (DNPR) maintains records of all hospitalizations in Denmark since 1977 [18]. Upon hospital discharge, the treating physician records a primary diagnosis describing the main reason for diagnostic work-up and treatment, and up to 19 secondary diagnoses describing comorbid conditions [18]. Diagnoses are coded according to the World Health Organization's International Classification of Diseases, 8th revision until the end of 1993 and 10th revision (ICD-10) thereafter. The treating physician also records discharge medication in the patient's electronic medical file and a copy is given to the patient and sent to his or her general practitioner.

\subsection{The Danish National Database of Reimbursed Prescriptions}

Danish pharmacies are required by law to register all prescriptions redeemed and BBs are prescription-only medication in Denmark. Electronic records of prescriptions redeemed since 2004 are kept in the Danish National Database of Reimbursed Prescriptions (NDP) [17]. A barcode identifier on each medication package enables automatic registration of information about the dispensed medication, including Anatomical Therapeutic Chemical (ATC) code, strength, pack size, pack quantity, and date of redemption. Information about the person redeeming the prescription, including civil registration number, is also registered.

\subsection{The Danish Civil Registration System}

All changes in vital status have been registered in the Danish Civil Registration System since 1968, with daily electronic updates [16]. Information about vital status is transferred from the Danish Civil Registration System to electronic medical patient files on a daily basis.

\subsection{Myocardial Infarction Patients}

Using primary and secondary in-patient hospital discharge diagnosis codes recorded in the DNPR, we identified patients who were hospitalized with MI in 2012 (ICD-10 code I21) without an antecedent MI diagnosis during 2006-2011. Eligible patients were discharged to their home from the Department of Cardiology, Aarhus University Hospital, Denmark, with BB treatment and redeemed one or more BB prescriptions within 6 months following hospital admission. Being highly specialized, the Department of Cardiology at Aarhus University Hospital treats cardiac patients admitted from home or transferred from other less specialized departments in the Central Denmark Region. 
Among 192 eligible patients, 100 were randomly selected and included in the study. Medical patient files were reviewed twice by one reviewer (SBP) to obtain information on gender, age, type and daily dose of $\mathrm{BBs}$ at discharge, and co-medication at discharge; that is, aspirin, statins, angiotensin-converting enzyme (ACE) inhibitors or angiotensin receptor blockers (ARBs), ticagrelor, clopidogrel, anticoagulants, and medications for diabetes mellitus. Using the NDP, we categorized patients as 'prior users' if they had redeemed one or more BB prescriptions prior to MI hospitalization. Information about vital status was also obtained by review of the electronic medical patient files (SBP).

\subsection{Beta-Blockers}

Information on all prescriptions for metoprolol, carvedilol, bisoprolol, atenolol, and propranolol redeemed during 2004-2013 was obtained for each patient from the NDP. Prescriptions redeemed on the same date were counted as one prescription. Because daily doses are not recorded directly in the NDP, they were computed for patients who redeemed two or more prescriptions as the content of each prescription (i.e., strength $\times$ pack size $\times$ pack quantity) divided by the number of days to the following prescription redemption [19]. A new daily dose was computed every time a prescription was redeemed. The daily dose was assumed to be constant between two prescription redemptions. The final daily dose in the study period was computed as the content of the before-last prescription redeemed, divided by the number of days between the before-last and the last prescription redeemed. No daily dose could be calculated for patients who redeemed only one prescription after discharge. When a patient changed $\mathrm{BB}$ type during follow-up, the last daily dose before the BB change was discarded and daily doses were not computed after a change of BB. Daily BB doses were recalculated to percentages of target dose; that is, daily $\mathrm{BB}$ dose/BB target dose. We defined four dose groups: $\leq 12.5 \%$ of target dose, $>12.5-25 \%$ of target dose, $>25-50 \%$ of target dose, and $>50 \%$ of target dose [13]. Dose calculations and ATC codes are provided in Table S1 in the electronic supplementary material.

\subsection{Statistical Analysis}

We categorized patients at discharge according to gender, age ( $<65$ years, $65-75$ years, or $>75$ years), BB type (metoprolol, carvedilol, bisoprolol, atenolol, or propranolol), BB dose group, and co-medication, overall and for prior users and new users, respectively. Median duration of hospitalization and age at discharge were computed with interquartile ranges (IQRs). We ascertained the total number of prescriptions redeemed during follow-up and the median number of prescriptions redeemed per patient. The median time from discharge to prescription redemption was computed.

The number of patients in each BB dose group at discharge and during the study period was ascertained. Each patient's final daily dose was compared with the dose at discharge and the proportion of patients who remained in the same BB dose group, or moved to a higher or lower dose group, respectively, was computed. To assess interdose variability during the study period, the difference between each daily dose and the preceding daily dose was computed, and the median variability for all patients was derived. The first daily dose was compared with the dose at discharge. The proportion of patients who changed $\mathrm{BB}$ dose group (any change, increase, or decrease) was ascertained at each prescription redemption.

\section{Results}

\subsection{Patient Demographics and Medication at Discharge}

We reviewed 106 eligible patient files. Six of them were excluded due to ambiguous BB discharge doses in the medical files. Included patients had a median hospitalization duration of 3 days (IQR 2-4). Table 1 shows demographics and medication at discharge. Median age at discharge was 70 years (IQR 60-78) for all patients, 67 years (IQR 55-76) for new users, and 74 years (IQR 64-79) for prior users. The majority of patients were discharged with metoprolol (Table 1). The median daily dose at discharge was $25.0 \%$ (IQR 25.0-25.0) of target dose for all patients, $25.0 \%$ (12.5-25.0) for new users and 25.0\% (IQR 25.0-50.0) for prior users. The median daily dose at discharge did not differ between men (25.0\% [IQR 25.0-25.0] of target dose) and women (25.0\% [IQR 12.5-37.5] of target dose). Only $8 \%$ of all patients were discharged with a daily BB dose $>50 \%$ of target dose, new users almost ten times less often than prior users, and $80 \%$ of patients were discharged with a daily BB dose $\leq 25 \%$ of target dose. Co-medication with statins and ticagrelor was more frequent among new users, while ACE inhibitors/ARBs and clopidogrel were more frequent among prior users.

\subsection{Prescription Redemptions}

A total of 688 prescriptions were redeemed during a median study period (i.e. time from discharge to last prescription redemption) of 400 days (IQR 318-486). The median number of prescriptions redeemed during the study period was 6 (IQR 4-8) per patient. Four patients changed BB type during the study period. 
Table 1 Patient demographics and medications at discharge after myocardial infarction

\begin{tabular}{|c|c|c|c|}
\hline & $\begin{array}{l}\text { All patients, } n(\%) \\
N=100\end{array}$ & $\begin{array}{l}\text { New users, } n(\%) \\
N=58\end{array}$ & $\begin{array}{l}\text { Prior users, } n(\%) \\
N=42\end{array}$ \\
\hline \multicolumn{4}{|l|}{ Gender } \\
\hline Male & $76(76.0)$ & 45 (77.6) & $31(73.8)$ \\
\hline Female & $24(24.0)$ & $13(22.4)$ & $11(26.2)$ \\
\hline \multicolumn{4}{|l|}{ Age, years } \\
\hline$<65$ & $34(34.0)$ & $23(39.7)$ & $11(26.2)$ \\
\hline $65-75$ & $31(31.0)$ & $18(31.0)$ & $13(31.0)$ \\
\hline$>75$ & $35(35.0)$ & $17(29.3)$ & $18(42.9)$ \\
\hline \multicolumn{4}{|l|}{ BB type } \\
\hline Metoprolol & $84(84.0)$ & $48(82.8)$ & $36(85.7)$ \\
\hline Carvedilol & $11(11.0)$ & $9(15.5)$ & $2(4.8)$ \\
\hline Bisoprolol & $4(4.0)$ & $1(1.7)$ & $3(7.1)$ \\
\hline Atenolol & $1(1.0)$ & $0(0.0)$ & $1(2.4)$ \\
\hline Propranolol & $0(0.0)$ & $0(0.0)$ & $0(0.0)$ \\
\hline \multicolumn{4}{|l|}{ BB dose group, $\%$} \\
\hline$\leq 12.5$ & $21(21.0)$ & $16(27.6)$ & $5(11.9)$ \\
\hline$>12.5-25.0$ & $59(59.0)$ & $38(65.5)$ & $21(50.0)$ \\
\hline$>25.0-50.0$ & $12(12.0)$ & $3(5.2)$ & $9(21.4)$ \\
\hline$>50.0$ & $8(8.0)$ & $1(1.7)$ & 7 (16.7) \\
\hline \multicolumn{4}{|l|}{ Co-medication } \\
\hline Aspirin & $98(98.0)$ & $58(100.0)$ & $40(95.2)$ \\
\hline Statin & $85(85.0)$ & $52(89.7)$ & $33(78.6)$ \\
\hline ACE-inhibitor/ARB & $50(50.0)$ & $25(43.1)$ & $25(59.5)$ \\
\hline Ticagrelor & $66(66.0)$ & $43(74.1)$ & $23(54.8)$ \\
\hline Clopidogrel & $27(27.0)$ & $10(17.2)$ & $17(40.5)$ \\
\hline Anticoagulant & $8(8.0)$ & $2(3.4)$ & $6(14.3)$ \\
\hline Diabetes medication & $8(8.0)$ & $6(10.3)$ & $2(4.8)$ \\
\hline
\end{tabular}

$A C E$ angiotensin-converting enzyme, $A R B$ angiotensin receptor blocker, $B B$ beta-blocker
Figure 1 illustrates time from discharge to prescription redemption. The median time from discharge to first prescription redemption was 2 days (IQR 1-4), longer for prior users (8 days [IQR 2-58]) than for new users (1 day [IQR 0-2]). Overall, the median time between prescription redemptions was 52 days (IQR 27-94) and the median time from discharge to the before-last prescription redemption, used for calculation of the final daily dose, was 321 days (IQR 227-391). Seven patients, among them five new users, redeemed only one prescription after discharge (median discharge dose $=12.5 \%$ of target dose [IQR 12.5-18.8]).

\subsection{Beta-Blocker Dose after Discharge}

Figure 2 illustrates the distribution of patients in each of the four BB dose groups at and following discharge. While the number of patients with a daily dose $\leq 25 \%$ of target dose decreased from 80 at discharge to 16 at first prescription redemption, the number of patients with a daily dose $>50 \%$ of target dose increased from 8 to 34. Still, only $31.5 \%(n=29)$ of patients had a final daily dose $>50 \%$ of target dose; that is, $19.0 \%(n=11)$ of new users and $42.9 \%(n=18)$ of prior users. The median final daily dose was $28.6 \%$ (IQR 22.2-66.1) of target dose, ranging from $24.3 \%$ (IQR 19.4-46.7) for new users to $43.1 \%$ (IQR 26.2-101.1) for prior users. Comparing the final daily dose with the dose at discharge, $40.2 \%(n=37$ [24 new users and 13 prior users]) remained in the same dose group (median dose change $-5.7 \%$ [IQR -18.0 to $4.2])$, while $50.0 \%(n=46$ [23 new users and 23 prior users]) moved to a higher dose group (median dose increase $89.6 \%$ [IQR 28.6-319.5]) and $9.8 \%(n=9$ [6 new users and 3 prior users]) moved to a lower dose group (median dose decrease $-51.5 \%$ [IQR-57.1 to -50.2$]$ ).

\subsection{Death During Follow-Up}

Two of the seven patients who redeemed only one prescription after discharge died during the study period, 13 and 41 days after prescription redemption, respectively. Both patients were men of at least 80 years and prior users. 
Fig. 1 Time from discharge to prescription redemption. Boxes illustrate medians with upper and lower quartiles. Whiskers illustrate maximum and minimum

Fig. 2 Distribution of patients in beta-blocker dose groups according to prescription number. Prescription no. 0 corresponds to discharge

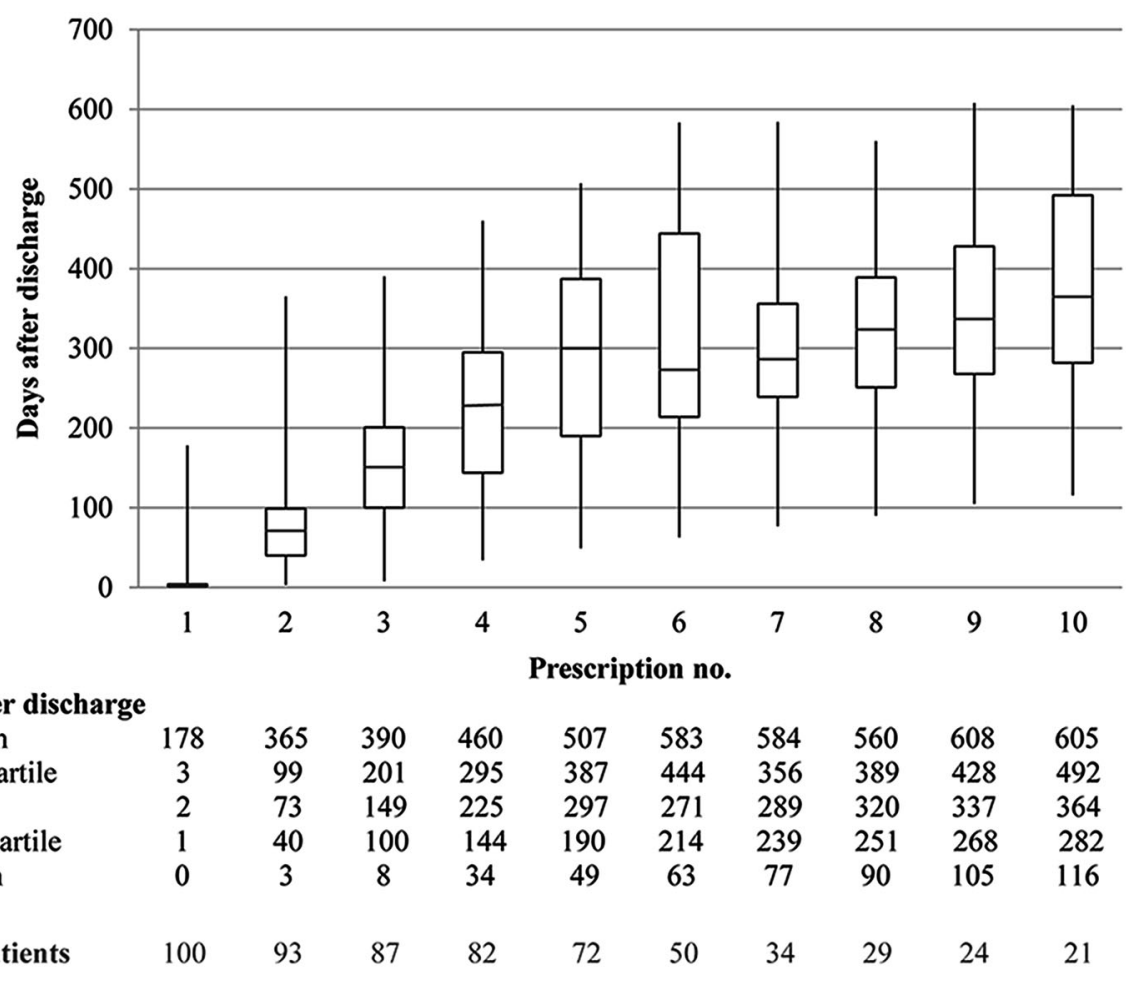

$\begin{array}{lcccccccccc}\text { Days after discharge } & & & & & & & & & \\ \text { Maximum } & 178 & 365 & 390 & 460 & 507 & 583 & 584 & 560 & 608 & 605 \\ \text { Upper quartile } & 3 & 99 & 201 & 295 & 387 & 444 & 356 & 389 & 428 & 492 \\ \text { Median } & 2 & 73 & 149 & 225 & 297 & 271 & 289 & 320 & 337 & 364 \\ \text { Lower quartile } & 1 & 40 & 100 & 144 & 190 & 214 & 239 & 251 & 268 & 282 \\ \text { Minimum } & 0 & 3 & 8 & 34 & 49 & 63 & 77 & 90 & 105 & 116 \\ \text { No. of patients } & 100 & 93 & 87 & 82 & 72 & 50 & 34 & 29 & 24 & 21\end{array}$

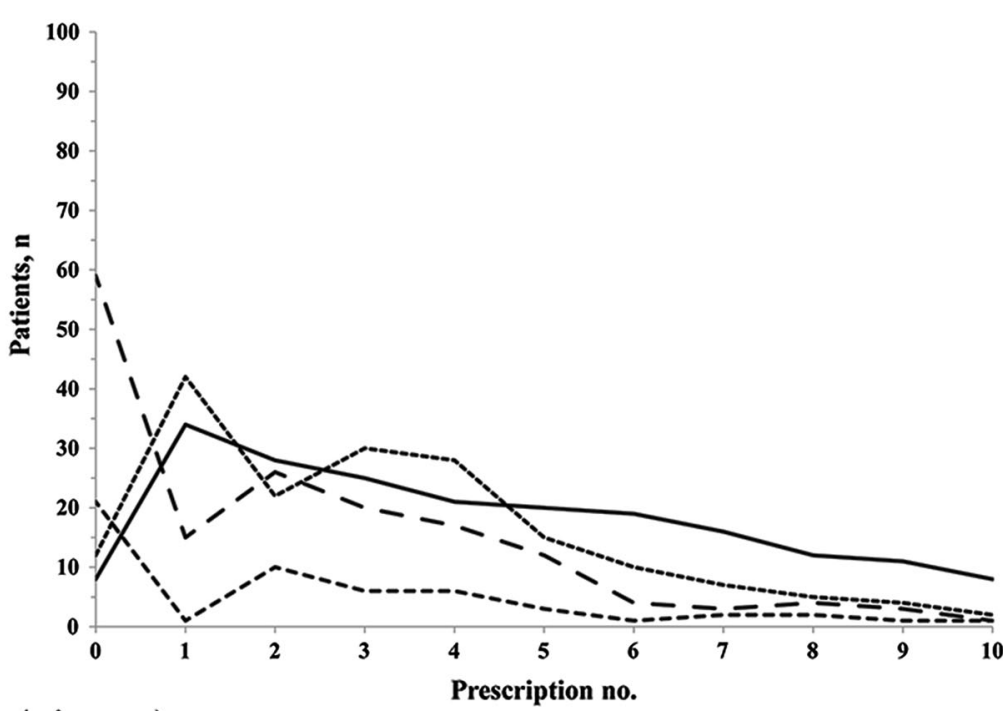

Dose group, $n$ (new/prior users)

\begin{tabular}{|c|c|c|c|c|c|c|c|c|c|c|c|}
\hline$\leq 12.5 \%-$ & $21(16 / 5)$ & $1(1 / 0)$ & $10(10 / 0)$ & $6(5 / 1)$ & $6(6 / 0)$ & $3(3 / 0)$ & $1(1 / 0)$ & $2(2 / 0)$ & $2(2 / 0)$ & $1(1 / 0)$ & $1(1 / 0)$ \\
\hline$>12.5-25 \%-$ & $59(38 / 21)$ & $15(10 / 5)$ & $26(14 / 12)$ & $20(14 / 6)$ & $17(13 / 4)$ & $12(9 / 3)$ & $4(4 / 0)$ & $3(2 / 1)$ & $4(2 / 2)$ & $3(3 / 0)$ & $1(0 / 1)$ \\
\hline$>25-50 \%$ & $12(3 / 9)$ & $42(26 / 16)$ & $22(16 / 6)$ & $30(19 / 11)$ & $28(14 / 14)$ & $15(11 / 4)$ & $10(5 / 5)$ & $7(4 / 3)$ & $5(2 / 3)$ & $4(2 / 2)$ & $2(2 / 0)$ \\
\hline$>\mathbf{5 0} \%$ & $8(1 / 7)$ & $34(16 / 18)$ & $28(11 / 17)$ & $25(9 / 16)$ & $21(8 / 13)$ & $20(7 / 13)$ & $19(9 / 10)$ & $16(6 / 10)$ & $12(4 / 8)$ & $11(3 / 8)$ & $8(3 / 5)$ \\
\hline N (new/prio & $100(58 / 42)$ & $92(53 / 39)$ & $86(51 / 35)$ & $81(47 / 34)$ & 4) $72(41 / 31$ & $50(30 / 20)$ & 0) $34(19 / 19$ & 9) $28(14 / 14$ & 4) $23(10 / 13$ & 3) $19(9 / 10)$ & $12(6 / 6)$ \\
\hline
\end{tabular}

They were discharged with metoprolol $25 \mathrm{mg}$ daily. As they only redeemed one prescription after discharge, no daily dose could be computed, but both patients picked up BB pills with the same strength (metoprolol $25 \mathrm{mg}$ ) as at their last prescription redemption before admission for MI. Another five patients with at least two BB prescription redemptions after discharge died during follow-up, between 227 and 400 days following discharge. Their age 
ranged from 70.8 to 90.1 years, three were women, and one was a prior user. All had redeemed three or more BB prescriptions after discharge. The latest computed daily dose was $\leq 12.5 \%$ of target dose for one patient, $12.5-25 \%$ of target dose for two patients, and $>50 \%$ of target dose for two patients. No daily dose could be computed after the last prescription redemption, but all five patients picked up BB pills with the same strength in $\mathrm{mg}$ as at the preceding prescription redemption, and four of the five also picked up the same number of pills as at the preceding prescription redemption. Of the 93 patients alive at the end of 2013, $26(28.0 \%)$ had not redeemed a BB prescription within the last 100 days.

\subsection{Inter-Dose Variability}

Figure 3 illustrates inter-dose variability during the study period. The largest dose change was observed when the first daily dose was compared with the dose at discharge. Comparing subsequent daily doses, there was less interdose variability as most median changes ranged within $\pm 10 \%$. Among prior users, daily doses fluctuated less than among new users (Figure S1 in the electronic supplementary material). From discharge to the final daily dose, we observed a median dose change of $11.1 \%$ (IQR -10.1 to 94.2), ranging from $6.4 \%$ (IQR -13.8 to 88.7 ) among new users to $11.1 \%$ (IQR -1.0 to 142.0 ) among prior users.

More than $70 \%$ of patients in our study changed BB dose group at first prescription redemption, particularly new users (Fig. 4a). The proportion of patients who changed BB dose group generally decreased with increasing prescription number (Fig. 4a). While the proportion of patients who moved to a higher $\mathrm{BB}$ dose group was larger at first prescription redemption than at subsequent redemptions (Fig. 4b), the proportion that moved to a lower $\mathrm{BB}$ dose group was largest at second prescription redemption (Fig. 4c).

\section{Discussion}

\subsection{Main Findings}

We found that only $8 \%$ of MI patients discharged from hospital with a BB were prescribed a daily dose $>50 \%$ of the target dose, while $80 \%$ were prescribed a daily dose $\leq 25 \%$ of the target dose. Although a considerable dose up-titration followed early after discharge, $40 \%$ of patients remained in the same $\mathrm{BB}$ dose group when the final daily dose was compared with the dose at discharge, and only $31.5 \%$ had a final daily dose $>50 \%$ of target dose. There was substantial attrition in BB prescription redemption (and presumably use) so that $28.0 \%$ of patients alive by the end of the study period had not redeemed a BB prescription within the last 100 days.

\subsection{Discharge Dosing}

To our knowledge, this is the first study of BB dosing patterns at and following discharge after MI in Denmark. Our results are in agreement with findings from other
Fig. 3 Dose variability during the study period for all patients, comparing each daily dose to the preceding daily dose. The first daily dose was compared with the dose at discharge

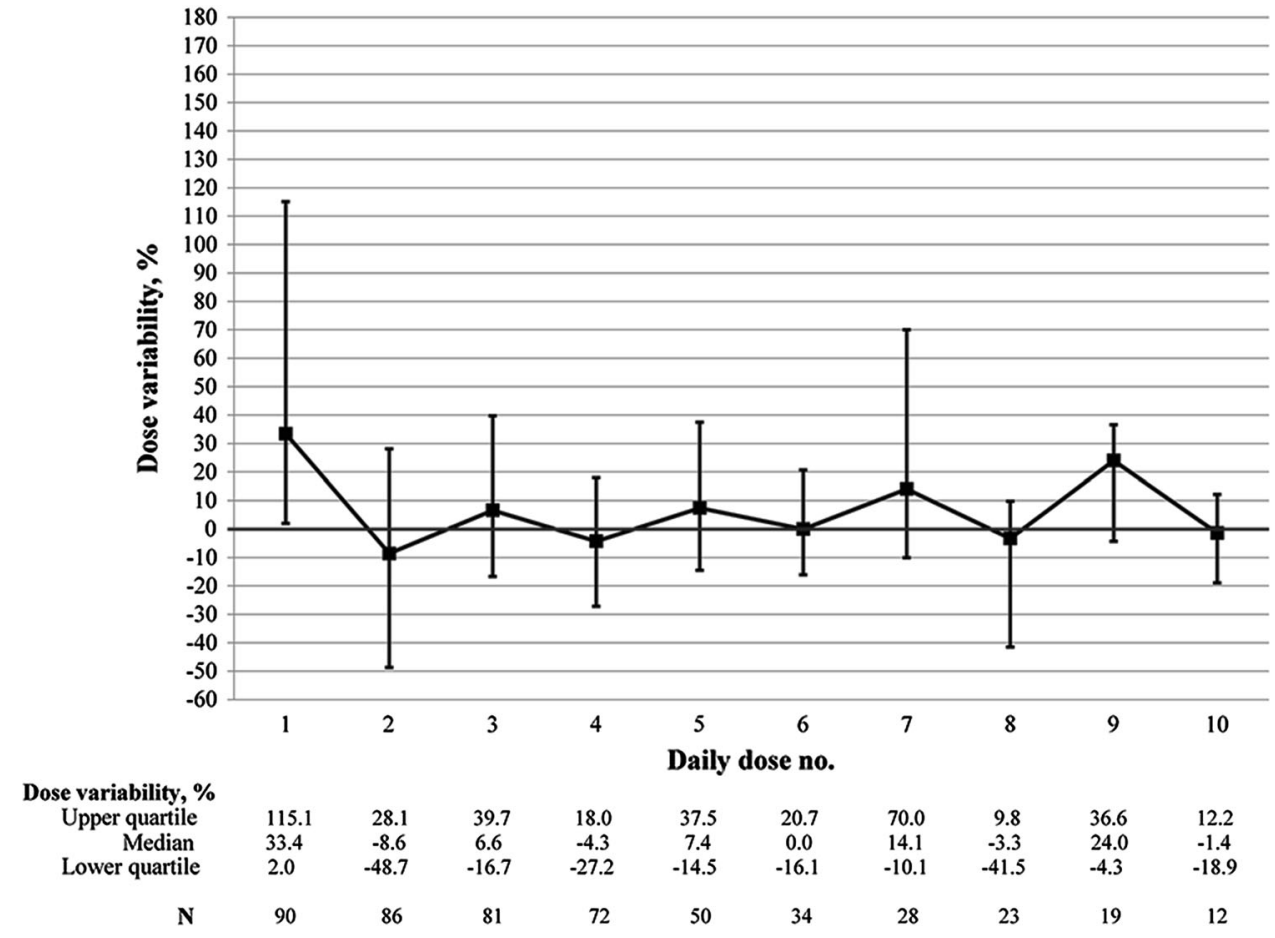


Fig. 4 Proportion of patients with any change (a), increase (b), or decrease (c) in BB dose group according to number of redeemed prescriptions. $B B$ beta-blocker
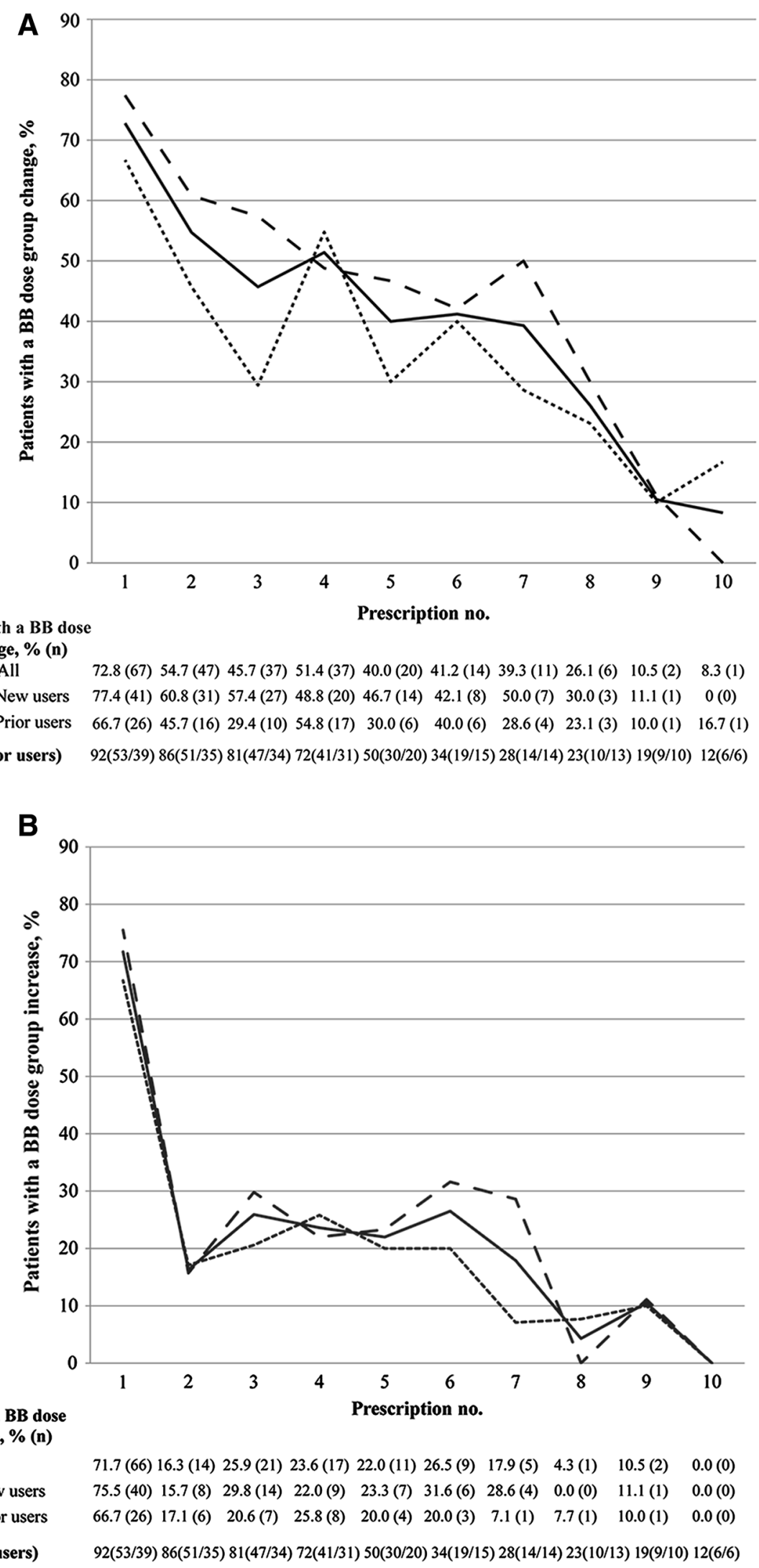
Fig. 4 continued

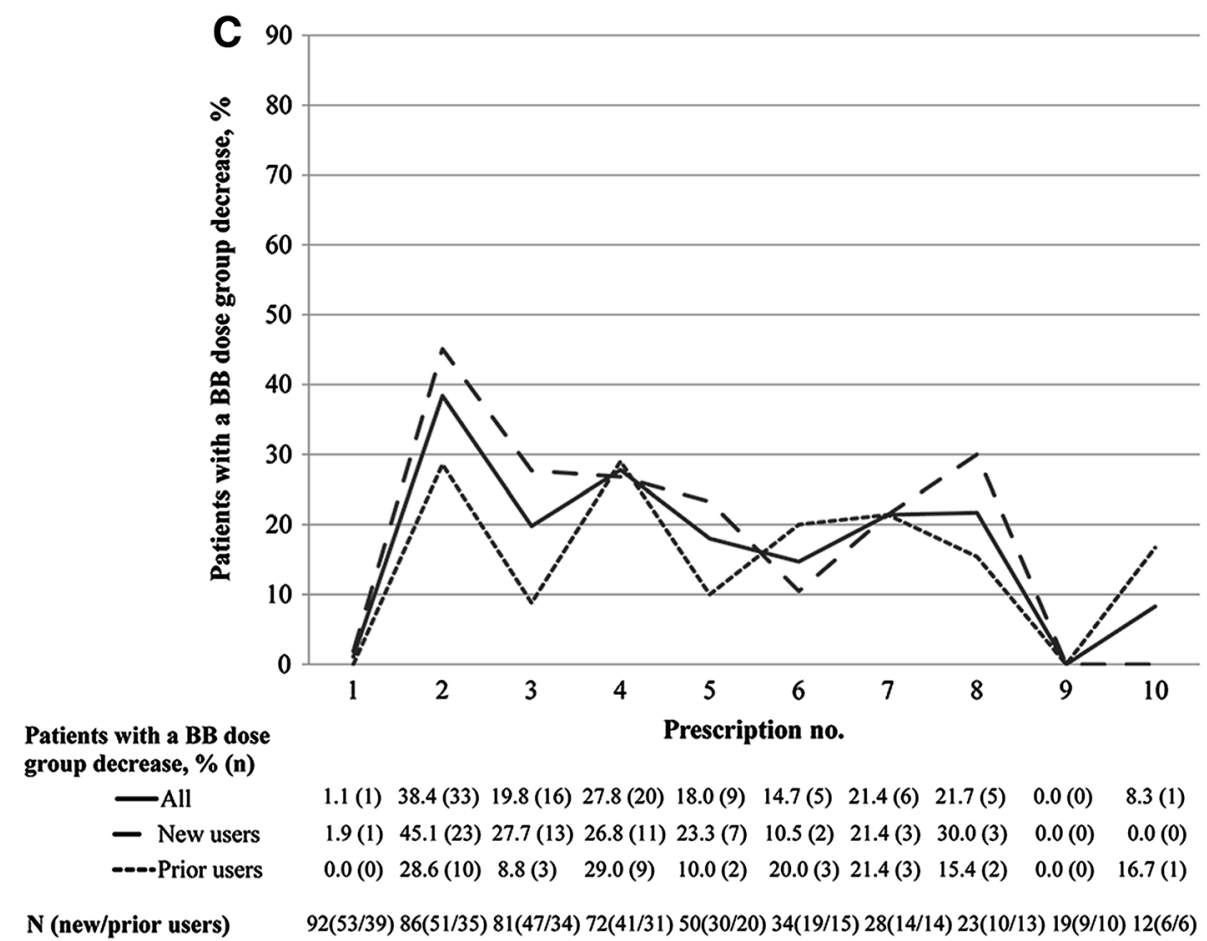

countries that have reported discharge doses $>50 \%$ of target doses among 13-60\% of post-MI patients [11-13]. These findings suggest that there is a widespread preference to use BBs at doses substantially below the target doses used in the clinical trials that established their efficacy. This practice has little supporting data except for the recently reported OBTAIN study [13].

\subsection{Dose Up-Titration and Achievement of Target Dose}

More than $70 \%$ of patients in our study changed BB dose group at first prescription redemption. In comparison, only $23.5 \%$ of post-MI patients treated with BBs in a US study had a dose change within 3 weeks of discharge [11]. This international difference may in part reflect that only $8 \%$ of patients in our study were discharged with daily BB doses $>50 \%$ of target dose, as opposed to $17 \%$ in the US study, causing physician propensity to up-titrate BB treatment among patients in our study to be higher. Furthermore, international differences in medication payment regulations, post-MI rehabilitation, and early follow-up may have played a role, as medical expenses [14, 15], participation in rehabilitation [20], and early follow-up [21] affect medication adherence.

Despite early dose up-titration, only $31.5 \%$ had a final daily BB dose $>50 \%$ of target dose. This finding is in line with that of Arnold et al., who reported that approximately $28 \%$ of 6748 MI patients had BB doses $>50 \%$ of target doses at 1-year follow-up [12]. Although our estimate may not be entirely comparable to these findings because final daily doses in our study were not necessarily achieved at 1-year follow-up, both studies indicate that the majority of MI patients do not achieve BB target dose approximately 1 year after discharge. Based on the high numbers of patients achieving BB target doses in clinical trials [11], it seems unlikely that BB intolerance prevented dose up-titration in all cases of under-dosing in our study.

\subsection{Treatment Discontinuation}

An important finding in our study was that $28.0 \%$ of patients alive at the end of the study period had not redeemed a $\mathrm{BB}$ prescription within the last 100 days and therefore likely disrupted BB treatment. This finding supports that of a US study in which approximately $30 \%$ of post-MI patients were not taking any BB at 12-month follow-up [12]. This is a significant clinical issue given the heavy evidence favoring any $\mathrm{BB}$ dose over no BB dose treatment following MI. We cannot know whether the seven patients who died during follow-up in our study had stopped taking BB. However, they all picked up the same or a larger amount of BB at their last prescription redemption as at the preceding prescription redemption, which may indicate continued use of the same or a higher daily dose.

\subsection{Dose Variability}

Although $40 \%$ had not changed BB dose group when the final daily dose was compared with the dose at discharge, a 
considerable proportion of patients changed BB dose group during the study period. This could reflect the use of arbitrary cut-off points to define BB dose groups, as even a small dose change could have caused movement to another BB dose group. Furthermore, some inter-dose variability is likely an artifact of the variability in time of filling prescription renewals. In a study of 55,315 Danish MI patients, Gislason et al. found that average daily BB doses 1-5 years after MI changed only 4-7\% compared with average doses of the first year after MI [19]. However, the authors did not include discharge doses and dose variability within the first year of discharge after MI. Our study provides new information about $\mathrm{BB}$ dosing at discharge and dose variability early after MI in Denmark.

\subsection{Study Strengths and Limitations}

The major advantages of this study lie in the completeness and accuracy of the nationwide prescription data used [17] and in the high validity of the MI diagnosis in the DNPR [22]. By using prescription data rather than questionnaires or medical patient files, we avoided recall bias and bias from primary non-adherence (i.e., failure to redeem prescriptions) [15]. Although we cannot completely exclude confounding from secondary non-adherence (i.e., failure to take the obtained medication [15]), the assumption remains reasonable that patients fill the prescription only when they need additional medication, indicating completion of the prior prescription. Thus, prescription data represent the best available estimate of actual BB medication use rather than intended use based on the prescribed dose. However, it should be noted that the daily doses derived from NDP data serve only as an approximation of the real dose as patients may not always redeem a new prescription on the day they run out of medication. A longer follow-up period would have strengthened our study. However, clinical trials have demonstrated that both short- and long-term BB treatment following MI reduce mortality significantly [1]. Finally, due to its purely descriptive design, no sample size calculation was performed for this study and the sample size was limited by the need for review of individual medical patient files. Thus, it was not possible to include all MI patients in Denmark and our data represent the BB use pattern from only one hospital in Denmark. Conceivable regional and global variations require further examination.

\section{Conclusions}

We found that BB treatment infrequently achieves target dose at discharge after MI. Despite dose up-titration early after discharge, most patients did not receive BB treatment in target dose approximately 1 year after MI. As dose may affect outcome [13], our findings provide important quality metrics and emphasize the importance of tracking BB use following MI to properly assess the impact of treatment and dose on survival.

Acknowledgments We thank Henriette Holmberg, Department of Cardiology, Aarhus University Hospital, and Jiaxin Chen, Department of Clinical Epidemiology, Aarhus University Hospital, for their assistance with the data collection for this study.

\section{Compliance with Ethical Standards}

Conflict of interest Dr Pedersen is supported by grants from the Danish Heart Foundation, Copenhagen, Denmark [Grant Number 12-04-R90-A3919-22728]; Aarhus University, Aarhus, Denmark; and the Health Research Foundation of the Central Denmark Region, Viborg, Denmark. Dr Goldberger has received support from Grant \#5U01HL080416 from the National Heart, Lung, and Blood Institute of the National Institutes of Health. Drs Nielsen and Bøtker declare that they have no conflict of interest. None of the funding sources had any role in the design, data collection, analysis, interpretation, or reporting of the study, nor in the decision to submit the article for publication.

Statement of human rights For this type of study, formal consent is not required.

Open Access This article is distributed under the terms of the Creative Commons Attribution-NonCommercial 4.0 International License (http://creativecommons.org/licenses/by-nc/4.0/), which permits any noncommercial use, distribution, and reproduction in any medium, provided you give appropriate credit to the original author(s) and the source, provide a link to the Creative Commons license, and indicate if changes were made.

\section{References}

1. Teo KK, Yusuf S, Furberg CD. Effects of prophylactic antiarrhythmic drug therapy in acute myocardial infarction. An overview of results from randomized controlled trials. JAMA. 1993;270:1589-95.

2. Freemantle N, Cleland J, Young P, Mason J, Harrison J. Beta blockade after myocardial infarction: systematic review and meta regression analysis. BMJ. 1999;318:1730-7.

3. Huang BT, Huang FY, Zuo ZL, et al. Meta-analysis of relation between oral $\beta$-blocker therapy and outcomes in patients with acute myocardial infarction who underwent percutaneous coronary intervention. Am J Cardiol. 2015;115:1529-38.

4. O'Gara PT, Kushner FG, Ascheim DD, et al. 2013 ACCF/AHA guideline for the management of ST-elevation myocardial infarction: executive summary: a report of the American College of Cardiology Foundation/American Heart Association Task Force on Practice Guidelines. Circulation. 2013;127:529-55.

5. Amsterdam EA, Wenger NK, Brindis RG, et al. 2014 AHA/ACC guideline for the management of patients with non-ST-elevation acute coronary syndromes: executive summary: a report of the American College of Cardiology/American Heart Association Task Force on Practice Guidelines. Circulation. 2014;130:2354-94.

6. Hjalmarson A, Elmfeldt D, Herlitz J, et al. Effect on mortality of metoprolol in acute myocardial infarction. A double-blind randomized trial. Lancet. 1981;2:823-7. 
7. Dargie HJ. Effect of carvedilol on outcome after myocardial infarction in patients with left-ventricular dysfunction: the CAPRICORN randomised trial. Lancet. 2001;357:1385-90.

8. CIBIS-II Investigators and Committees. The Cardiac Insufficiency Bisoprolol Study II (CIBIS II): a randomized trial. Lancet. 1999;353:9-13.

9. Viskin S, Kitzis I, Lev E, et al. Treatment with beta-adrenergic blocking agents after myocardial infarction: from randomized trials to clinical practice. J Am Coll Cardiol. 1995;25:1327-32.

10. Beta-blocker Heart Attack Trial Research Group. A randomized trial of propranolol in patients with acute myocardial infarction. I. Mortality results. JAMA. 1982;247:1707-14.

11. Goldberger JJ, Bonow RO, Cuffe M, et al. Beta-blocker use following myocardial infarction: low prevalence of evidencebased dosing. Am Heart J. 2010;160:435-42.

12. Arnold SV, Spertus JA, Masoudi FA, et al. Beyond medication prescription as performance measures: optimal secondary prevention medication dosing after acute myocardial infarction. J Am Coll Cardiol. 2013;62:1791-801.

13. Goldberger JJ, Bonow RO, Cuffe M, et al. Effect of beta-blocker dose on survival after acute myocardial infarction. J Am Coll Cardiol. 2015;66:1431-41.

14. Mathews R, Peterson ED, Honeycutt E, et al. Early medication nonadherence after acute myocardial infarction: insights into actionable opportunities from the TRreatment with ADP receptor iNhibitorS: Longitudinal Assessment of Treatment patterns and Events after Acute Coronary Syndrome (TRANSLATE-ACS) Study. Circ Cardiovasc Qual Outcomes. 2015;8:347-56.
15. Kolandaivelu K, Leiden BB, O'Gara PT, Bhatt DL. Non-adherence to cardiovascular medications. Eur Heart J. 2014;35:3267-76.

16. Schmidt M, Pedersen L, Sørensen HT. The Danish Civil Registration System as a tool in epidemiology. Eur J Epidemiol. 2014;29:541-9.

17. Johannesdottir SA, Horváth-Puhó E, Ehrenstein V, Schmidt M, Pedersen L, Sørensen HT. Existing data sources for clinical epidemiology: the Danish National Database of Reimbursed Prescriptions. Clin Epidemiol. 2012;4:303-13.

18. Schmidt M, Schmidt SA, Sandegaard JL, Ehrenstein V, Pedersen L, Sørensen HT. The Danish National Patient Registry: a review of content, data quality, and research potential. Clin Epidemiol. 2015;7:449-90.

19. Gislason GH, Rasmussen JN, Abildstrøm SZ, et al. Long-term compliance with beta-blockers, angiotensin-converting enzyme inhibitors, and statins after acute myocardial infarction. Eur Heart J. 2006;27:1153-8.

20. Shah ND, Dunlay SM, Ting HH, et al. Long-term medication adherence after myocardial infarction: experience of a community. Am J Med. 2009;122:961.e7-13.

21. Daugherty SL, Ho PM, Spertus JA, et al. Association of early follow-up after acute myocardial infarction with higher rates of medication use. Arch Intern Med. 2008;168:485-91.

22. Madsen M, Davidsen M, Rasmussen S, Abildstrom SZ, Osler M. The validity of the diagnosis of acute myocardial infarction in routine statistics: a comparison of mortality and hospital discharge data with the Danish MONICA registry. J Clin Epidemiol. 2003;56:124-30. 